\title{
TRANSESOPHAGEAL ECHOCARDIOGRAPHIC AND CLINICAL FEATURES OF AORTIC INTRAMURAL HEMATOMA
}

Kevin M. Harris, MD $^{\mathrm{a}}$

Alan C. Braverman, $\mathrm{MD}^{\mathrm{a}}$

Fernando R. Gutierrez, MD ${ }^{\mathrm{b}}$

Benico Barzilai, $\mathrm{MD}^{\mathrm{a}}$

Víctor G. Dávila-Román, $\mathrm{MD}^{a}$
Objective: This study sought to determine the transesophageal echocardiographic features and natural history of patients with aortic intramural hematoma. Methods: The transesophageal echocardiograms of all patients who had symptoms indicative of aortic dissection over 6 years were reviewed. Measurements were made of the involved aortic segment in the study patients, and follow-up was obtained. Results: In patients with aortic intramural hematoma, the wall thickness of the involved segment was significantly greater for descending segments than ascending segments (ascending aorta $7 \pm 2 \mathrm{~mm}$, descending aorta $15 \pm 6 \mathrm{~mm}, p=0.0016$ ). In each case, the crescent-shaped intramural hematoma involved one wall predominantly, leading to compression of the aortic lumen. The findings of echolucent areas and displaced intimal calcium were found in the majority of patients. Four of eight patients with intramural hematoma of the ascending aorta were treated medically and four were treated surgically. The 30-day mortality was $50 \%$ in the medically treated patients and $0 \%$ in the surgically treated group. Four of 11 patients with isolated intramural hematoma of the descending aorta were treated medically and seven were treated surgically. All medically treated and $86 \%$ of surgically treated patients were alive at $\mathbf{3 0}$ days. Conclusions: Aortic intramural hematoma has distinct and identifiable transesophageal echocardiographic features. These data support those of previous studies documenting high morbidity and mortality in patients with aortic intramural hematoma. ( $J$ Thorac Cardiovasc Surg 1997;114:619-26)
A ortic dissection is a life-threatening disorder associated with a high mortality. The recognition of classic aortic dissection by imaging techniques requires the identification of the intimal flap that results from blood dissecting between the intimal and medial layers of the aortic wall. ${ }^{1}$ Noninvasive imaging techniques such as computed tomography (CT), magnetic reso-

From the Cardiovascular Division ${ }^{\mathrm{a}}$ and the Mallinckrodt Institute of Radiology, Washington University School of Medicine, St. Louis, Mo.

Supported in part by a Minority Scientist Development Award from the American Heart Association, Dallas, Texas, to Dr. Dávila-Román.

Received for publication Oct. 31, 1996; revisions requested May 12, 1997; revisions received June 5, 1997; accepted for publication June 9, 1997.

Address for reprints: Kevin M. Harris, MD, Cardiovascular Division, Washington University School of Medicine, 660 South Euclid Ave., Box 8086, St. Louis, MO 63110.

Copyright $(1997$ by Mosby-Year Book, Inc.

$0022-5223 / 97 \$ 5.00+0 \quad \mathbf{1 2 / 1 / 8 3 7 8 9}$ nance imaging (MRI), and transesophageal echocardiography (TEE) have recently been used to identify a variant of classic aortic dissection, aortic intramural hematoma (IMH). ${ }^{2-5}$ Aortic IMH was first described in 1920 by Krukenberg ${ }^{6}$ and is characterized by the presence of thrombosis between the intimal and medial vessel layers, giving the aortic wall an appearance of focal thickening. Patients with aortic IMH have symptoms similar to those associated with classic aortic dissection, but the characteristic intimal flap is not visible by imaging techniques. Studies to date have suggested that aortic IMH carries morbidity and mortality rates that are similar to those of classic aortic dissection. ${ }^{2-5}$ Thus early recognition and treatment of patients with aortic IMH is important to optimize outcomes.

TEE allows for rapid, accurate bedside evaluation of patients with classic aortic dissection, ${ }^{7-10}$ and it has been suggested that it would be equally useful in the identification of patients with aortic IMH. Thus the purpose of this study was to define 


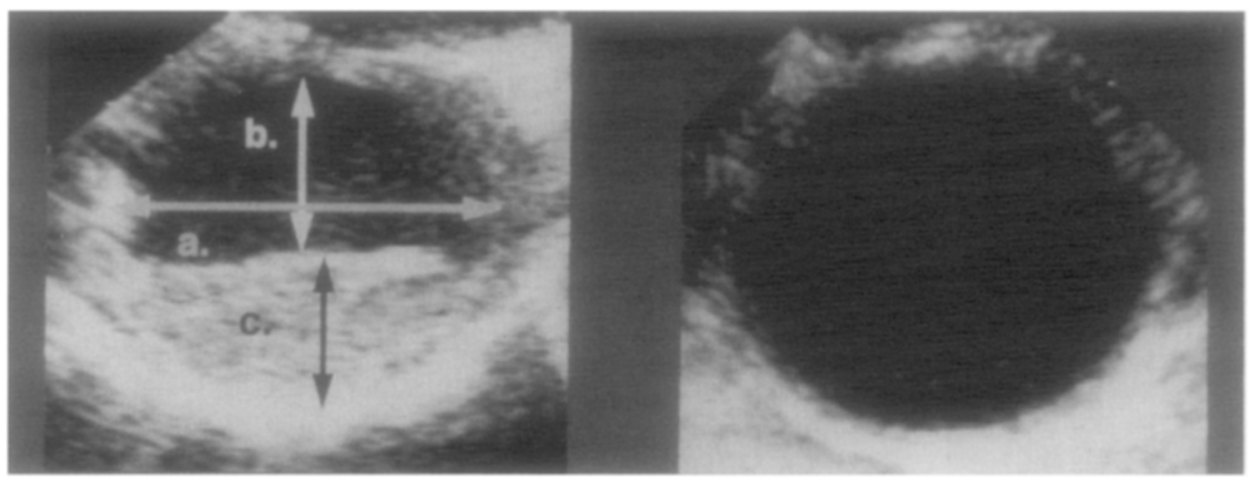

Fig. 1. Short-axis TEE view of descending aorta at site of measurements. Left, TEE from patient with IMH. Right, Normal aorta. Aortic lumen measurements are indicated in major-axis diameter (a) and minor-axis diameter $(b)$. Aortic wall thickness is indicated by $c$.

the echocardiographic features of aortic IMH and characterize the natural history of patients with this disorder.

\section{Methods}

Patient population. The studies of all patients undergoing TEE for aortic dissection at Barnes-Jewish Hospital at Washington University Medical Center in St. Louis over a 6-year interval (from January 1990 to January 1996) were reviewed retrospectively. A total of 84 patients had aortic dissection; 65 had classic aortic dissection and 19 had aortic IMH. A region of focal wall thickening with a thrombus-like consistency and no intimal flap identified aortic IMH. ${ }^{5}$ Patients were included in the study if the diagnosis was suspected on the basis of TEE and subsequently confirmed at the time of the operation, by CT, by MRI, or by clinical course (1 patient). Patients with hematoma resulting from trauma were excluded. In four patients IMH involved the ascending aorta alone, in 11 the descending aorta alone, and in four the hematoma involved both the ascending and the descending aorta. One patient with IMH has been previously reported (N Engl J Med 1997;336:1875).

For comparison, the TEEs from 10 consecutive agematched patients who underwent the study for indications other than suspected thoracic aortic disease were reviewed. Each patient had extensive study of the aorta. The indications for the study included evaluation for suspected endocarditis in four, valvular disease in four, cardiac source of embolus in one, and evaluation of left ventricular function in one patient.

Clinical criteria. Clinical data including coronary risk factors, symptoms, cardiovascular findings on physical examination, information gathered with other imaging modalities, intraoperative reports, and/or surgical pathology findings were obtained by review of medical records and interview with the cardiac surgeon. Follow-up data were obtained by telephone after hospital discharge.

TEE. TEE was performed by use of commercially available monoplane, biplane, or multiplane transesophageal transducers (Hewlett-Packard, Andover, Mass., and Acuson Computed Sonography, Mountain View, Calif.).
Excellent two-dimensional images of the thoracic aorta (ascending and descending aorta), the left and right ventricles, the pericardium, and the cardiac valves and color-flow Doppler imaging to evaluate for aortic regurgitation were available in every case.

Analysis of TEE images. Images of a total of 23 aortic segments (8 ascending and 15 descending aorta segments) containing an aortic IMH were analyzed. Measurements were made by use of an off-line image analysis system (Nova Microsonics, Indianapolis, Ind.). Aortic IMH was identified as localized thickening of the aortic wall that had a thrombus-like appearance and was measured in a short-axis projection at the site of maximal involvement, as shown in Fig. 1. In the short-axis view, measurements were made of the involved and the uninvolved portion because aortic IMH involves only a portion of the aortic circumference. A normalized wall thickness ratio was derived by comparing involved and uninvolved segments of aorta. Compression of the normal circular aortic lumen by the $\mathrm{IMH}$ results in two aortic diameters defined as the major axis (largest) and minor axis (smallest), respectively, and these were measured from trailing edge to leading edge (Fig. 1). An aortic lumen major/minor axis ratio was derived from these measurements.

TEE images were examined for the presence or absence of aortic atherosclerotic plaques, ${ }^{11-14}$ intimal calcium, displacement of calcium, and echolucent areas, which represent pools of blood within the aortic wall. Examples of TEE features are demonstrated in Fig. 2. The presence or absence of complications of aortic dissection, such as pleural effusion, mediastinal hemorrhage, moderate or severe aortic insufficiency, or a large pericardial effusion, was also noted.

Confirmatory imaging studies or surgical findings. Images of the involved aortic segment obtained by alternative methods were reviewed by an experienced cardiovascular radiologist who was blinded to the TEE findings or the clinical course. On noncontrast CT, the aortic IMH was identified as a high-density rim of the involved portion of the aortic wall in cases in which the study was performed early after the initial bleeding episode. This hemorrhage can then be seen extending medially and distally. Displacement of intimal calcification is another 

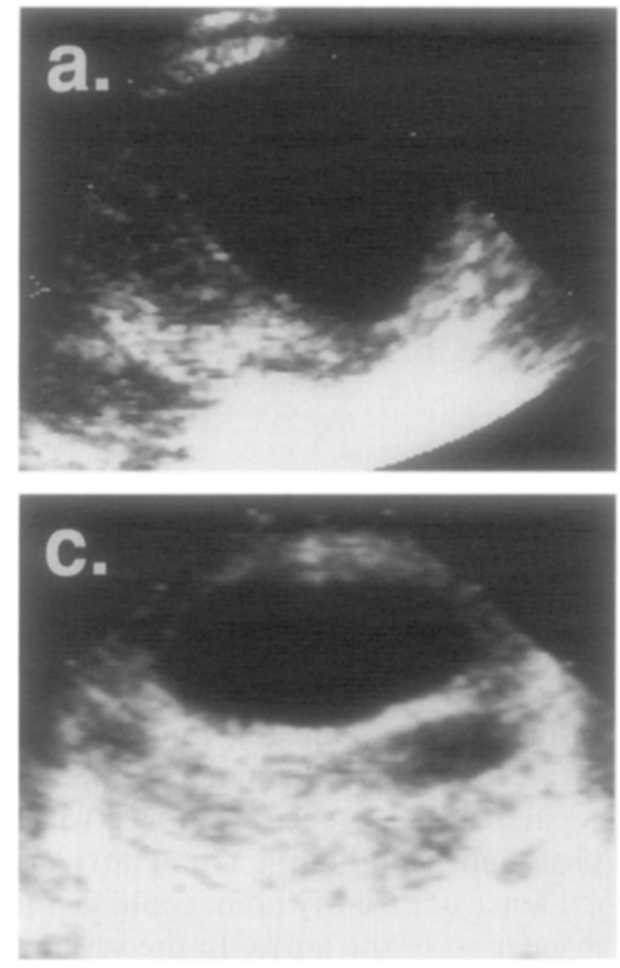
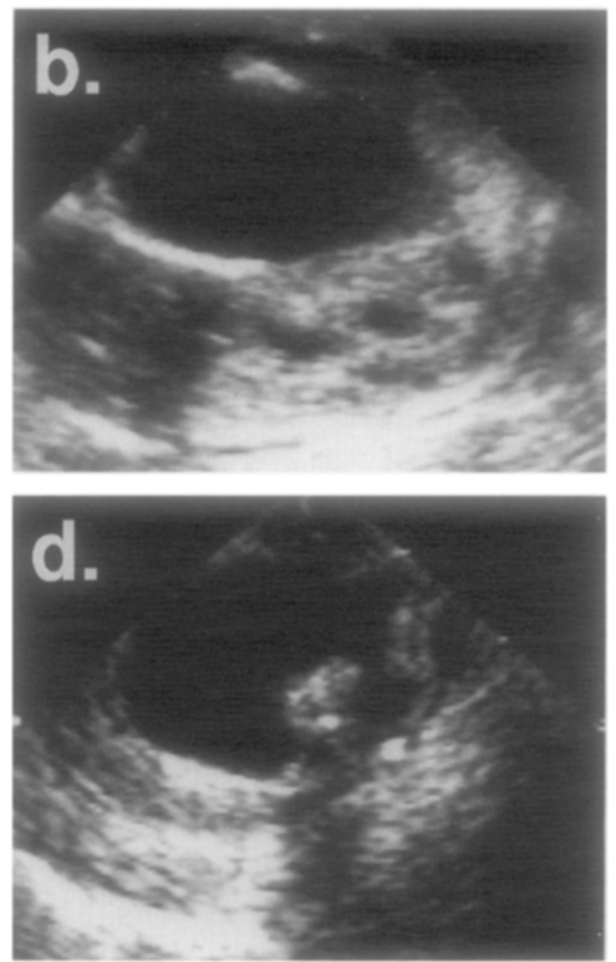

Fig. 2. TEE features of patients with IMH. a, IMH of descending aorta. There is marked focal wall thickening $(1.4 \mathrm{~cm})$, and wall thickness ratio is $4.7 . \mathrm{b}$ and $\mathrm{c}$, Eccentric IMH causes crescent-shaped deformity of aortic lumen. Aortic lumen major/minor axis ratio is 1.6 in $\mathbf{b}$. Note echolucent areas within the hematoma and displaced intimal calcium in $\mathbf{b}$ and $\mathbf{c}$. $\mathbf{d}$, Atherosclerotic plaque associated with an ulcer and IMH.

distinguishing feature. Contrast enhancement was used to determine the degree of encroachment on the aortic lumen. ${ }^{15}$ As with CT, MRI depicts the thickened aortic wall resulting from the IMH. Signal intensity characteristics are variable and will generally depend on the age of the hematoma. In most cases, T1-weighted images demonstrate hyperintense foci consistent with subacute intramural hemorrhage within the aortic wall. ${ }^{16}$ In 11 of 19 $(58 \%)$ patients, the diagnosis of aortic IMH was confirmed by findings during the operation and pathologic results as well.

Statistical analysis. Measurements were expressed as mean \pm standard deviation. Differences between study and control patients were evaluated with the use of the unpaired $t$ test or Fisher's exact test when appropriate.

\section{Results}

Patient characteristics. The study population consisted of 12 women and seven men with a mean age of $70.9 \pm 10.0$ years. All patients had symptoms consistent with aortic dissection, including chest pain in seven, back pain in seven, chest and back pain in four, and a sense of impending doom in one. The symptoms had been present for less than 24 hours in 11 patients, for 1 to 7 days in six patients, and for more than 7 days in two patients. Sixteen patients had a history of hypertension or were hypertensive on admission, nine had a smoking history, six had known coronary disease, and five had known hypercholesterolemia. No patient had a blood pressure discrepancy greater than $10 \mathrm{~mm} \mathrm{Hg}$ between the two arms. On the admission chest x-ray film, 12 patients had a widened mediastinum or dilated thoracic aorta and five of 11 patients with isolated descending aortic IMH had a left pleural effusion.

Echocardiographic characteristics. The TEE findings in patients with aortic IMH are listed in Table I. The wall thickness of descending segments containing IMH was significantly greater than that of ascending segments with IMH $(15 \pm 6 \mathrm{~mm}$ vs $7 \pm$ $2 \mathrm{~mm}, p=0.0016$ ). Inasmuch as the IMH involves primarily one wall, the mean wall thickness ratio was $4.5 \pm 1.6$. The wall thickening led to compression of the normal circular lumen; thus the aortic lumen major/minor axis ratio was $1.3 \pm 0.2$. 
Table I. TEE findings in patients with IMH

\begin{tabular}{lccc}
\hline & Mean & Range & 95\% Confidence limits \\
\hline Wall thickness, ascending aorta $(\mathrm{mm})$ & $7 \pm 2$ & $4-11$ & $5.3-8.9$ \\
Wall thickness, descending aorta $(\mathrm{mm})$ & $15 \pm 6$ & $8-25$ & $12.0-18.2$ \\
Aortic lumen, ascending aorta $(\mathrm{mm})$ & $39 \pm 7$ & $29-48$ & $33.4-44.8$ \\
Aortic lumen, descending aorta $(\mathrm{mm})$ & $31 \pm 6$ & $20-41$ & $26.9-34.4$ \\
Wall thickness ratio & $4.5 \pm 1.6$ & $2-7.7$ & $3.5-5.5$ \\
Aortic lumen ratio & $1.3 \pm .2$ & $1.1-1.7$ & $1.2-1.4$ \\
Echolucent arcas & & $19 / 23=83 \%$ & $61 \%-95 \%$ \\
Displaced intimal calcium & & $66 \%-97 \%$ \\
\hline
\end{tabular}

Echolucent areas were present in 19 of the 23 segments involved by IMH, and intimal calcium was displaced in 20 of $23 \mathrm{IMH}$ segments. Atherosclerotic plaques at the site of the hematoma were noted in $42 \%$ of ascending segments and $100 \%$ of the descending segments involved with IMH.

The age-matched control patients had a mean age of $70.4 \pm 7$ years. The mean wall thickness in the control patients measured $3 \pm 1 \mathrm{~mm}$ in the ascending aorta and $4 \pm 2 \mathrm{~mm}$ in the descending aorta; thus the corresponding IMH segments were significantly thicker ( $p<0.0001, p<0.0001$, respectively). The aortic lumen of patients with IMH was greater than that found in control patients (ascending aorta $32 \pm 4 \mathrm{~mm}, p=0.01$; descending aorta $23 \pm 3 \mathrm{~mm}$, $p=0.002$ ). Control patients had a wall thickness ratio of $1.1 \pm 0.4$ and an aortic lumen major/minor axis ratio of $1.1 \pm 0.1$, which differed from that of the patients with IMH $(p<0.0001, p=0.001)$. No control patient was noted to have echolucent areas or displaced intimal calcium $(p<0.0001, p<$ 0.0001 ). Atherosclerotic plaques were noted in $33 \%$ of ascending segments and in $70 \%$ of descending segments in the control patients.

Six of eight patients $(75 \%)$ with IMH of the ascending aorta had large pericardial effusions and/or cardiac tamponade, and seven of eight patients $(88 \%)$ had moderate or severe aortic insufficiency. Mediastinal hematoma suggestive of aortic rupture was noted in three of 11 patients with isolated IMH of the descending aorta.

Cardiovascular imaging and/or surgical findings. Imaging of the thoracic aorta with CT (11 patients) and/or MRI ( 7 patients) confirmed the presence of aortic IMH in all patients. Representative TEE and MRI findings in two patients with aortic IMH can be seen in Figs. 3 and 4. Aortography was performed in eight patients, but the diagnosis of aortic IMH was suspected with this technique in only three $(38 \%)$, whereas the other five aortograms showed no ab- normalities. Thus aortography has a low yield in this condition.

Surgical exploration was performed in 11 patients and intraoperative findings and gross pathologic inspection consistently showed thrombus within the aortic media, confirming the diagnosis of aortic IMH. Four patients underwent surgical repair of the ascending aorta with the use of profound hypothermic circulatory arrest, and no evidence of an entry point could be found on close inspection of the aorta. In the seven patients with IMH of the descending aorta, an atherosclerotic ulcerated plaque or small intimal communication (decompression rent) was identified. These were not believed to be entry points as found in true aortic dissection. Surgical findings confirmed rupture into the pericardium in five patients. Only one patient underwent no additional imaging or surgical procedure. This patient had symptoms consistent with an acute aortic dissection and shock. The TEE study showed IMH of the ascending aorta and cardiac tamponade. Emergency pericardiocentesis revealed hemorrhagic pericardial fluid, but the patient was not believed to be a surgical candidate and died within hours.

Clinical course. Eight patients had IMH of the ascending aorta; four were treated medically and four underwent surgery (Table II). The 30-day mortality for the four patients treated medically was $50 \%$. One of the patients treated medically died of unrelated causes 1 year later; the other is alive at 1 year of follow-up. All four patients who underwent surgery were alive at 30 days with a mean follow-up of $16 \pm 14$ months. All four patients with involvement of the ascending and descending aorta also had arch involvement; two were treated medically and two underwent surgical replacement of the ascending aorta to the level of the arch.

Among patients with isolated IMH of the descending aorta, seven required surgical intervention: 


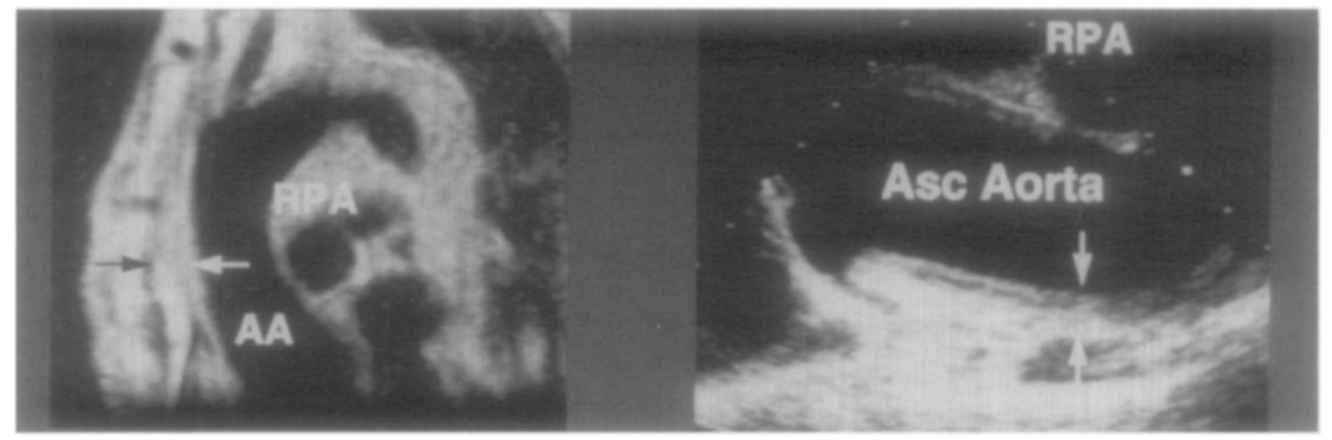

Fig. 3. Corresponding MRI (sagittal view) (left) and TEE (longitudinal view) (right) features in a patient with involvement of the ascending aorta with IMH. Involvement of the anterior wall of the ascending aorta is noted by arrows. $A A$, Ascending aorta; $R P A$, right pulmonary artery.

three for rupture, two for expansion with pain, and two for persistent pain. Six of seven were alive at 30 days with a mean follow-up of $12 \pm 15$ months. All four medically treated patients with isolated descending aortic IMH were alive at 30 days and at long-term follow-up a mean $24 \pm 22$ months after diagnosis. Three of the medically treated patients had repeat imaging a mean of 17 months after diagnosis, which documented regression of IMH in all cases.

\section{Discussion}

Aortic IMH can be identified by TEE with a high degree of accuracy. Echocardiographic indicators of IMH include focal aortic wall thickening, an eccentric aortic lumen, displaced intimal calcium, and areas of echolucency within the aortic wall. Our results support those of previous studies that show that aortic IMH results in high mortality in patients treated medically. ${ }^{2-5}$

Echocardiographic criteria. The echocardiographic features of IMH were first described by MohrKahaly and associates. ${ }^{2}$ They found that in three patients with ascending aortic IMH and in 12 with descending aortic IMH, a wall thickness of greater than $0.7 \mathrm{~cm}$ that extended longitudinally from 3 to $20 \mathrm{~cm}$ was indicative of hematoma. ${ }^{2}$ The present study, to our knowledge the largest echocardiographic series to date, shows that descending aortic segments were markedly thicker than ascending segments. Thus, whereas IMH of the descending aorta is easily recognized as marked thickening of an eccentric segment, ascending aortic IMH may be characterized by lesser degrees of thickening. We found echolucent areas in $83 \%$ of our patients with aortic IMH and in none of our control patients. Atherosclerotic plaques were noted in $42 \%$ of our patients with ascending aortic IMH and in all patients with descending aortic IMH. These findings are similar to the findings of the earlier series, in which echolucent areas were seen in $67 \%$ of patients and atherosclerotic plaques were noted in $73 \%$ of the patients. ${ }^{2}$

Our data add further descriptive findings that may be useful in the diagnosis of aortic IMH. We noted that the crescent-shaped IMH narrows the aortic lumen to a significant degree, leading to an increased aortic lumen major/minor axis ratio. We also found that the intimal calcium was displaced medially in $87 \%$ of our patients having a hematoma. Although no single echocardiographic finding is an absolute indicator of aortic IMH, the presence of multiple features is highly suggestive of the diagnosis.

Differentiation from other conditions. The echocardiographic characteristics of classic aortic dissection, such as intimal flap and entry point, are widely recognized. However, the diagnosis of aortic IMH may be difficult at times, inasmuch as this entity resembles other pathologic entities involving the aorta, such as atherosclerotic disease and aneurysmal dilatation with intraluminal thrombi. The distinction between severe aortic atherosclerosis and aortic IMH may be difficult. ${ }^{2}$ Distinguishing aortic IMH from the other pathologic conditions depends on identification of the intima, which is often calcified and therefore has a bright echodense appearance. Thickening beneath the intima is suggestive of IMH; thickening above the intima represents a different pathologic condition, such as occurs with thrombus within the aorta (Fig. 5).

Atherosclerotic disease of the aorta, which is prevalent in elderly persons, is characterized by focal or generalized thickening of the aortic wall 


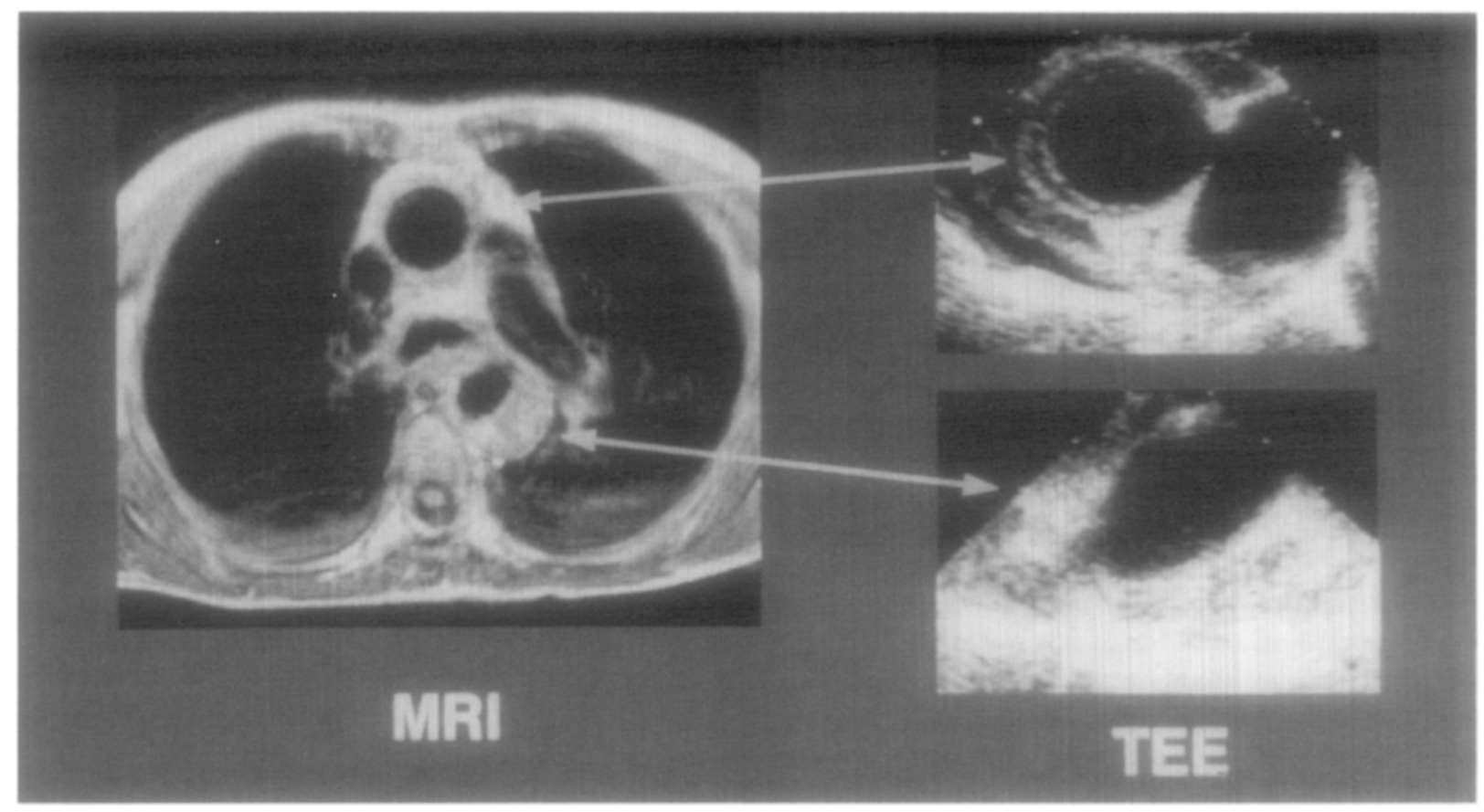

Fig. 4. MRI (left) and TEE (right) images of a patient with involvement of both the ascending and descending aorta. Upper right TEE image shows focal thickening of ascending aorta. Lower right images show crescent-shaped IMH (which is thicker than ascending segment) compressing the aortic lumen.

leading to irregularities on the intimal surface, calcification, and thickening of the adjoining intima. ${ }^{11-14}$ One echocardiographic feature pointing to the diagnosis of aortic IMH as opposed to atherosclerosis is a crescent-shaped thickening of the aortic wall extending over a 5 to $20 \mathrm{~cm}$ length of the aorta. $^{2}$ In contrast to aortic atherosclerosis, IMH involves a defined segment of the wall (i.e., increased wall thickness ratio) and is associated with echolucent areas that represent a pool of blood within the hematoma. Thus differentiation of aortic IMH and atherosclerotic disease of the aorta can be achieved by careful evaluation of these echocardiographic features.

Pathogenesis. The pathogenesis of classic aortic dissection and more recently of IMH has been the subject of considerable debate. Most studies defining the pathologic characteristics of aortic dissection have noted that in $5 \%$ to $13 \%$ of patients the entry point of blood into the dissection cannot be visualized. ${ }^{17,17 a}$ However, others contend intimal tears can be found on close inspection. ${ }^{18}$ IMH has traditionally been considered to be a form of dissection without an entry point, although, as in classic dissection, close surgical inspection of patients with aortic IMH has been shown to reveal entry points. ${ }^{19}$
The incidence of aortic IMH in our study (23\%) and other aortic IMH series ${ }^{2,5}$ is similar to the incidence in patients in earlier series of classic dissection in which an entry point could not be identified. ${ }^{17,17 a}$ The four patients in our series with ascending aortic IMH who underwent surgery had no apparent entry point on surgical inspection, which lends support to the hypothesis that rupture of the vasa vasorum may cause ascending aortic IMH.

All of our patients with descending aortic IMH also had severe aortic atherosclerosis; in those undergoing surgical treatment, associated atherosclerotic ulcerated plaques were identified. ${ }^{20-22} \mathrm{Al}-$ though the role of atherosclerosis in classic aortic dissection is controversial, ${ }^{17,23}$ our data add support to findings of previous studies suggesting that atherosclerotic plaque rupture and/or penetrating ulcers may be associated with descending aortic IMH. $^{2}$

\section{Clinical features and natural history}

Risk factors. Our patients had risk factors for aortic IMH similar to those described by others, including old age and a history of hypertension., ${ }^{1,2}$ The prevalence of atherosclerosis in older patients may predispose them to formation of penetrating ulcers and localized hematoma instead of classic 
Table II. Clinical course of patients with IMH

\begin{tabular}{|c|c|}
\hline Treatment & Thirty-day mortality \\
\hline \multicolumn{2}{|c|}{ Ascending aorta IMH $(n=8)$} \\
\hline Medical & $2 / 4(50 \%)$ \\
\hline Surgical & $0 / 4(0 \%)$ \\
\hline \multicolumn{2}{|c|}{ Descending aorta $I M H(n=11)$} \\
\hline Medical & $0 / 4(0 \%)$ \\
\hline Surgical & $1 / 7(14 \%)$ \\
\hline
\end{tabular}

aortic dissection. Roberts ${ }^{23}$ has suggested that patients with atherosclerosis have limited dissection or an absence of longitudinal extension that leads to limitation of the extent of the dissection. The preponderance of women in our series is contrary to what has been reported in studies of patients with classic aortic dissection.

Ascending aorta. The complication rate and mortality in ascending aortic IMH are high, ${ }^{17 a, 24-27}$ and thus surgical intervention has been recommended. However, these recommendations are based on small numbers of patients. ${ }^{2-5,24-27}$ Including our eight patients, a total of 49 patients with ascending aortic IMH have been described to date..$^{2-5,24,25,27}$ Twenty-six $(53 \%)$ of these patients have been treated surgically and 23 medically. A strategy of initial medical management often leads to urgent surgical treatment. ${ }^{2,27}$ When patient data from these studies are pooled, survival of the medically treated patients is $43 \%$. $^{2-5,24,25}$

Inasmuch as the ascending aorta is enveloped by pericardium, extravasation of blood through the adventitia likely leads to the high incidence of effusions seen in our series $(75 \%)$ and is similar to that in other series. ${ }^{2,4}$ Because of the high morbidity and mortality associated with medical treatment, patients with ascending aortic IMH should undergo emergency surgical repair.

Descending aorta. Among the 11 patients with isolated IMH of the descending aorta, three $(27 \%)$ underwent surgical repair for rupture, two for expansion and continued pain, and two for persistent pain. The fact that the hemorrhage lies immediately adjacent to a thin layer of adventitia may help to explain the propensity to rupture. Four of our patients were treated medically and all have done well with an average follow-up of 24 months. As with classic descending aortic dissection, aggressive medical management (hypertension and pain control) and follow-up imaging is necessary. Of 53 patients with descending aortic

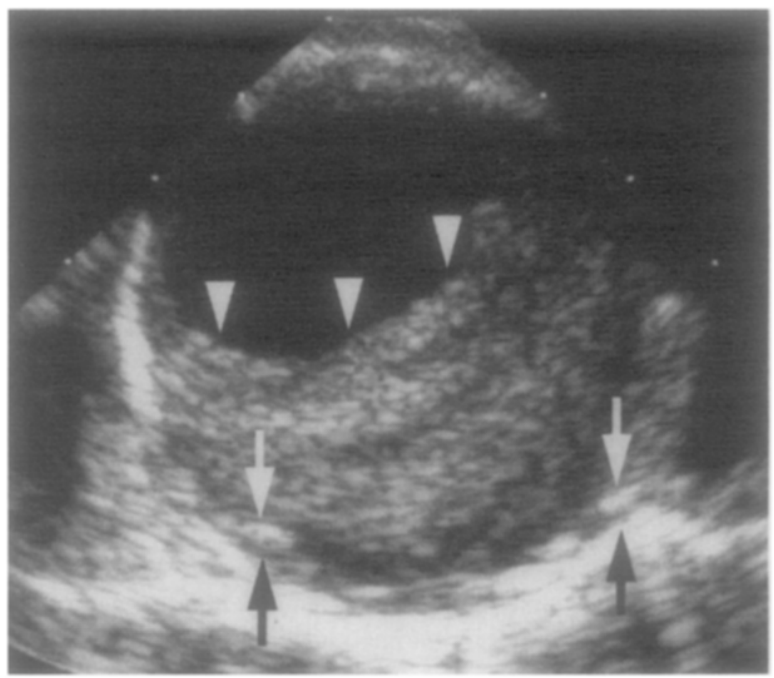

Fig. 5. Transverse TEE image of an intraluminal thrombus within an aneurysm in the descending thoracic aorta. In contrast to intramural hematoma (Figs. 1 to 4 ), note that the thrombus (arrowheads) lies superior to the intimal calcium (arrows).

IMH in our series and other series, 33 have received medical treatment and 20 underwent surgery with similar survival on follow-up $(91 \%$ and $80 \%$, respectively). ${ }^{2-5,24}$

Limitations of the study. This is a retrospective study and as such suffers from the inherent limitations of such studies, including evaluation bias and lack of standardized evaluation and treatment.

\section{Conclusions}

The use of noninvasive imaging techniques such as CT, MRI, and TEE has improved the accuracy of the diagnosis of classic aortic dissection, whose hallmark is the identification of an intimal flap. A variant of classic aortic dissection, aortic IMH has specific TEE features that differentiate it from other abnormalities of the thoracic aorta. These criteria can be applied prospectively to identify patients with this condition and to define the clinical features associated with it. Our results confirm those of previous studies that show a high mortality for this condition and support recommendations for rapid diagnosis and treatment, similar to that for classic aortic dissection.

We appreciate the contributions of Michael Rosenbloom, MD, Beth Engeszer (editorial assistance), and Linda Gallo (secretarial assistance). 


\section{REFERENCES}

1. Nienaber CA, von Kodolitsch Y, Nicolas V, Siglow V, Piepho $A$, Brockhoff $C$, et al. The diagnosis of thoracic aortic dissection by noninvasive imaging procedures. $\mathrm{N}$ Engl J Med 1993;328:1-9.

2. Mohr-Kahaly S, Erbel R, Kearney P, Puth M, Meyer J. Aortic intramural hemorrhage visualized by transesophageal echocardiography: findings and prognostic implications. J Am Coll Cardiol 1994;23:658-64.

3. Robbins RC, McManus RP, Mitchell RS, Latter DR, Moon $M R$, Olinger GN, et al. Management of patients with intramural hematoma of the thoracic aorta. Circulation 1993;88: $1-10$.

4. Yamada T, Tada S, Harada J. Aortic dissection without intimal rupture: diagnosis with MR imaging and CT. Radiology 1988;168:347-52.

5. Nienaber CA, von Kodolitsh $\mathrm{Y}$, Peterson B, Loose R, Helmchen A, Spielmann RP. Intramural hemorrhage of the aorta: diagnostic and therapeutic implications. Circulation 1995;92:1465-72.

6. Krukenberg E. Beitrage zur Frage des Aneurysma dissecans. Beitr Pathol Anat Allg Pathol 1920;67:329-51.

7. Cigarroa JE, Isselbacher EM, DeSanctis RW, Eagle KA. Diagnostic imaging in the evaluation of suspected aortic dissection. N Engl J Med 1993;328:35-43.

8. Erbel R, Borner N, Steller D, Brunier J, Thelen M, Pfeiffer $\mathrm{C}$, et al. Detection of aortic dissection by transesophageal echocardiography. Br Heart J 1987;58:45-51.

9. Erbel R, Daniel W, Visser C, Engberding R, Roelandt J, Rennollet H, et al. Echocardiography in diagnosis of aortic dissection. Lancet 1989;1:457-60.

10. Khandheria BK. Aortic dissection: the last frontier. Circulation 1993;87:1765-8.

11. Karalis DG, Chandrasekaran K, Victor MF, Ross JJ, Mintz GS. Recognition and embolic potential of intraaortic atherosclerotic debris. J Am Coll Cardiol 1991;17:73-8.

12. Katz ES, Tunick PA, Rusinek H, Ribakove G, Spencer FC, Kronzon I. Protruding aortic atheromas predict stroke in elderly patients undergoing cardiopulmonary by-pass: experience with intraoperative transesophageal echocardiography. J Am Coll Cardiol 1992;20:70-7.

13. Dávila-Román VG, Barzilai B, Wareing TH, Murphy SF, Schechtman KB, Kouchoukos NT. Atherosclerosis of the ascending aorta: prevalence and role as an independent predictor of cerebrovascular events in cardiac patients. Stroke 1994;25:2010-6.

14. Dávila-Román VG, Barzilai B, Wareing TH, Murphy SF,
Kouchoukos NT. Intraoperative ultrasonographic evaluation of the ascending aorta in 100 consecutive patients undergoing cardiac surgery. Circulation 1991;84(Suppl):III47-53.

15. Kazerooni EA, Bree RL, Williams DM. Penetrating atherosclerotic ulcers of the descending thoracic aorta: evaluation with CT and distinction from aortic dissection. Radiology 1992;183:759-66.

16. Yucel EK, Steinberg FL, Egglin TK, Geller SC, Waltman AC, Athanasoulis CA. Penetrating aortic ulcers: diagnosis with MR imaging. Radiology 1990;177:779-81.

17. Gore I. Pathogenesis of dissecting aneurysm of the aorta. In: AMA Arch Pathol 1952;53:142-53.

17a.Braverman AC, Harris KM. Management of aortic intramural hematoma. Curr Opin Cardiol 1995;10:501-4.

18. Larson EW, Edwards WD. Risk factors for aortic dissection: a necropsy study of 161 cases. Am J Cardiol 1984;53:849-55.

19. Borst HG, Heinemann MK, Stone CD. Pathogenesis. In: Surgical treatment of aortic dissection. Edinburgh: Churchill Livingstone; 1996. p. 47-54.

20. Stanson AW, Kazmier FJ, Hollier LH, Edwards WD, Pairolero $\mathrm{PC}$, Sheedy $\mathrm{PF}$, et al. Penetrating atherosclerotic ulcers of the thoracic aorta: natural history and clinicopathologic correlations. Ann Vasc Surg 1986;1:15-23.

21. Spittell PC, Spittell JA, Joyce JW, Tajik AJ, Edwards WD, Schaff HV, et al. Clinical features and differential diagnosis of aortic dissection: experience with 236 cases (1980 through 1990). Mayo Clin Proc 1993;68:642-51.

22. Braverman AC. Penetrating atherosclerotic ulcers of the aorta. Curr Opin Cardiol 1994;9:591-7.

23. Roberts WC. Aortic dissection: anatomy, consequences, and causes. Am Heart J 1981;101:195-214.

24. Alfonso F, Goicolea J, Aragoncillo P, Hernandez R, Macaya C. Diagnosis of aortic intramural hematoma by intravascular ultrasound imaging. Am J Cardiol 1995;76:735-8.

25. Vilacosta I, San Roman A, Peral V, Castillo J, Domínguez L, Batle E, et al. Imaging aortic intramural hematoma: identification of two groups of patients. Circulation 1995;92(Suppl): $\mathbf{1 3 0 7 .}$

26. O'Gara PT, DeSanctis RW. Acute aortic dissection and its variants toward a common diagnostic and therapeutic approach. Circulation 1995;92:1376-8.

27. Keren A, Kim CB, Hu BS, Eyngorina I, Billingham ME, Mitchell RS, et al. Accuracy of biplane and multiplane transesophageal echocardiography in diagnosis of typical acute aortic dissection and intramural hematoma. J Am Coll Cardiol 1996;28:627-36. 